\title{
Análisis estructural de la torre del homenaje de la Alhambra de Granada (España)
}

\section{Assessment of procedures for the structural analysis of "Homenaje Tower" in the Alhambra in Granada (Spain)}

D. Villegas ${ }^{(*)}$, M. Cámara ${ }^{(*)}, \underline{\text { V. Compán }}{ }^{(*)}$

\section{RESUMEN}

Este artículo pretende describir la respuesta estructural de la Torre del Homenaje, una de las torres del complejo de la Alhambra (Granada), y por otro lado revisar las distintas técnicas utilizadas en el análisis estructural de edificios históricos aplicándolas a dicho objeto para valorar el alcance de cada una de ellas.

La torre se analiza mediante tres procedimientos con diferentes niveles de complejidad. En primer lugar, se utiliza el método de líneas de empuje para valorar los diferentes coeficientes de seguridad desde un punto de vista de la estabilidad. En segundo lugar, se lleva a cabo un análisis elástico y lineal aplicando el método de los elementos finitos para identificar posibles concentraciones de tensiones de tracción.

Por último, se ha realizado un análisis estático no lineal tipo pushover sobre cinco modelos numéricos diferentes para valorar la respuesta estructural ante acciones horizontales de los muros perimetrales y de los pórticos interiores.

Palabras clave: Fábrica de ladrillo; análisis estructural; Alhambra; pushover.

\section{ABSTRACT}

This paper aim, on one hand, to describe the structural response of "Homenaje Tower", one of the towers of the Historical Complex of the Alhambra, in Granada (Spain) and, on the other hand, to look over the different techniques used in the structural analysis of historical buildings, applying them to the tower to assess the scope of each of them.

The tower is analyzed from three methods that count on different levels of complexity. First, an analysis using graphical method for limit analysis is carried out to assess the different safety coefficients from a stability point of view. Secondly, a finite element linear elastic analysis is applied in order to identify possible stress concentration zones, in particular zones with tensile stresses.

Finally, a nonlinear static pushover analysis has been carried out over five different numerical models to assess the structural response to horizontal loads of perimeter walls and interior porticos.

Keywords: Brick masonry; structural analysis; Alhambra; pushover.

${ }^{(*)}$ Universidad de Sevilla, España.

Persona de contacto/Corresponding author: compan@us.es (V. Compán)

Cómo citar este artículo/Citation: Villegas, D., Cámara, M., Compán V. (2014). Análisis estructural de la torre del homenaje de la Alhambra de Granada (España). Informes de la Construcción, 66(EXTRA-1): mo17, doi: http://dx.doi.org/10.3989/ic.13.101.

Licencia/License: Salvo indicación contraria, todos los contenidos de la edición electrónica de Informes de la Construcción se distribuyen bajo una licencia de uso y distribución Creative Commons Reconocimiento no Comercial 3.o. España (cc-by-nc). 


\section{INTRODUCCIÓN}

La Torre del Homenaje es uno de los objetos más elevados del complejo de la Alhambra de Granada. Fue la entrada primitiva a la Alcazaba y utilizada como vivienda. Se cree que en ella se estableció el fundador de la Alhambra, Al-Hamar (1).

Se trata de una torre tronco-piramidal que cuenta con seis niveles más el nivel de terraza y almenas. Con unas dimensiones en planta de unos 12,0 × 10,5 m y con 24,8 m de altura, se encuentra unida a la muralla perimetral del recinto en la esquina oeste y a lo largo de los dos niveles inferiores (Figura 1). El acceso a la torre se realiza directamente en el tercer nivel, desde la cumbre de la muralla y a través de un pasaje cubierto por una bóveda de cañón. De este nivel de acceso parte un núcleo vertical que comunica los niveles superiores.

A excepción del primer y el último nivel, todos se configuran en planta divididos en seis espacios cuadrados o sensiblemente rectangulares, dejando en la zona central dos pilares de gran dimensión que recorren el edificio en toda su altura. Dichos espacios están cubiertos por sistemas de bóvedas y cúpulas muy variados y rellenos hasta conformar el suelo del nivel inmediatamente superior.

En la configuración interior de la torre, pueden reconocerse algunas de las reglas empíricas propias de la época (2). Tanto las pilas principales como los arcos y bóvedas responden a proporciones que justifican el dimensionado de los mismos. Así, por ejemplo, el ancho de los estribos de las bóvedas es una fracción entera de la luz salvada (3).

Lo variado de las soluciones empleadas en el interior contrasta con la contundencia del aspecto exterior de la torre. En el exterior, las reglas empíricas dejan paso a espesores de mayor relevancia. El muro perimetral de la torre cuenta con espesores que van desde los 2,35 $\mathrm{m}$ en su base hasta los $0,80 \mathrm{~m}$

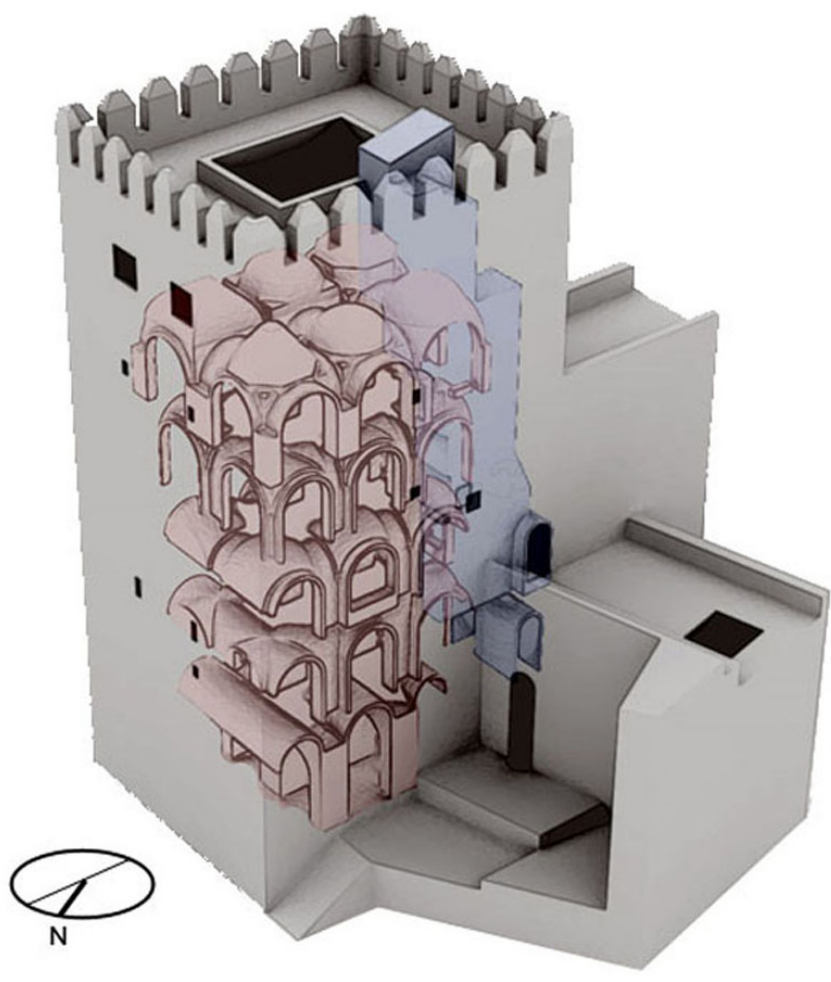

Figura 1. Torre del Homenaje. Modelo tridimensional. en el nivel superior, con la intención de dotar a la torre de una masa contundente propia del carácter defensivo de la edificación que además fuera eficaz ante la ya entonces conocida virulencia de los temblores de la zona.

El contraste descrito entre interior y exterior alcanza los materiales estructurales empleados. Así, los sistemas interiores junto con la cara interior del muro son de fábrica de ladrillo, mientras que el grueso del muro exterior es de tapial calicastrado.

\subsection{Actuaciones precedentes}

No existe constancia de que se hayan realizado intervenciones de consideración en los elementos sustentantes interiores. Asimismo, no existen registros de desplomes o colapsos locales interiores que hicieran pertinentes intervenciones de reconstrucción.

En cuanto a los elementos exteriores, tenemos constancia de dos intervenciones relevantes:

Por un lado, consta un derrumbe parcial del muro de la esquina suroeste de la torre (esquina donde confluyen la muralla oeste, la muralla sur y el núcleo de comunicación vertical). El derrumbe se atribuye con toda probabilidad a un terremoto registrado a mediados del siglo XVIII. La reconstrucción se realizó utilizando aparejo de ladrillo (diferenciándolo de los elementos de tapial del resto del muro) y se sustituyó la arista tronco-piramidal por una vertical pura.

Por otro lado, en el siglo XIX, se acometió una reconstrucción del nivel superior. Se reconstruyeron las almenas (dotándolas de una altura superior a las originales) y tres de las cuatro naves que conforman el patio de la planta sexta. En esta ocasión se sustituyó el sistema abovedado, del que aún queda constancia en una de las naves, por un sistema plano de entrevigado de madera (4).

\subsection{Estado actual}

Actualmente, el edificio presenta un buen estado de conservación, sin patologías reseñables de carácter activo. Las grietas y defectos reflejados en un informe realizado por el CEDEX en 1995 (5) no han sufrido ninguna evolución de consideración.

A partir del estado de agrietamiento general de la torre (presentándose la grieta más importante en la fachada este), y de los resultados del estudio detallado de las cotas de nivelación registradas en distintos puntos de todas las plantas (llevado a cabo por el CEDEX igualmente), se identifica un patrón general en el que es el ámbito $\mathrm{N}-\mathrm{O}$ el más deprimido, seguido de los ámbitos N-E y S-E, mientras que el S-O es el menos deprimido dada la mayor rigidez que aportan los adarves de la muralla que acomete.

\section{MODELOS Y PROCEDIMIENTOS DE ANÁLISIS ESTRUCTURAL}

\subsection{Hipótesis simplificativas}

Dado que uno de los objetos de este documento es la compresión global del comportamiento estructural de la torre, no se consideran las posibles alteraciones que en aspectos como la 
rigidez pudieran provocar los defectos con que actualmente cuenta la misma.

No se ha considerado la forma tronco-piramidal de la torre por consistir en una desviación mínima de los muros hacia el interior de aproximadamente $10 \mathrm{~cm}(5)$.

No se ha considerado, dentro de las condiciones de contorno, la vinculación de la torre con la muralla por varias razones. En primer lugar, un análisis constructivo de la unión torremurallas denota que la trabazón entre ambos elementos es, cuanto menos, dudosa. En segundo lugar, una vinculación firme posicionada en esquina como está (Figura 1) implicaría movimientos de rotación de la torre ante acciones horizontales. Sin embargo, y a pesar de las acciones sísmicas a que ha estado sometida la torre, no existen grietas que denoten este fenómeno, lo que refuerza la hipótesis de que la trabazón torre-murallas sea de escasa entidad.

En relación a las acciones, se han considerado las verticales derivadas del peso propio de los distintos elementos, así como una acción horizontal de presión actuando en la dirección más desfavorable. En una construcción pesada como la que nos ocupa, el peso propio es la acción gravitatoria más relevante. Por su parte, la acción horizontal es de valor incremental hasta la determinación de la capacidad resistente última de la estructura.

\subsection{Definición de modelos y procedimientos de análisis}

El primer tipo de análisis se lleva a cabo sobre el denominado Modelo 1. Se trata de un análisis estructural a partir de líneas de empuje (6) (7). Este procedimiento, muy empleado en análisis estructurales preliminares de estructuras históricas, permite tener una primera cuantificación a nivel isostático del estado general de la estructura a cargas gravitatorias (8) (9).

Así, se estudia la estabilidad global de los muros perimetrales y se cualifica el efecto de los empujes de los arcos interiores sobre ellos. Igualmente, se establece un coeficiente de seguridad geométrico de los elementos interiores relevantes, tales como los principales arcos de descarga y las cúpulas y bóvedas que cubren el interior.

Como segundo tipo de análisis, se plantea un análisis elástico y lineal utilizando el Método de los Elementos Finitos sobre el denominado Modelo 2. A pesar de que se trata de un análisis del que no se pretende obtener una historia de evolución de grietas, sí pueden extraerse datos orientativos que ayuden a entender el comportamiento general del edificio (10). El análisis se realiza teniendo en cuenta cargas gravitatorias exclusivamente y considerando distintas fases del proceso constructivo.

Por último, se plantea un análisis estático no lineal con un comportamiento inelástico del material mediante curvas de capacidad (11) con objeto de valorar la respuesta estructural del edificio ante acciones horizontales. Este procedimiento incorpora una simulación inelástica del material y es capaz de limitar las tensiones de tracción y de incorporar daño para degradar la rigidez de los elementos en el tramo post-fallo.

Este modelo de análisis, basado igualmente en el Método de los Elementos Finitos, se aplica a dos sub-modelos geométricos. El primero de ellos incluye un análisis plano, reali- zándose un estudio de capacidad de una de las secciones representativas del edificio (Modelo 3). Teniendo en cuenta las simplificaciones descritas anteriormente, la torre es sensiblemente simétrica en sus dos direcciones principales, por lo que es razonable estudiar algunos de sus parámetros mediante un modelo de deformación plana. Así, se desarrolla un modelo plano donde se reflejan todos los elementos estructurales incluidos en la sección principal del edificio, siendo especialmente interesante para estudiar con mayor profundidad todos los fenómenos locales que puedan producirse.

Por otro lado, se evalúa el efecto tridimensional a través de un modelo que contempla geometría y rigidez del muro perimetral, compuesto por tapial y ladrillo, como elemento predominante en la resistencia de los esfuerzos horizontales (Modelo 4).

Este último procedimiento de análisis, aplicado a los Modelos 3 y 4 , ha sido desarrollado mediante un análisis tipo pushover (12). Se trata de un proceso de análisis estáticos incrementales sucesivos. Se efectúa incrementando una carga lateral proporcional a los desplazamientos modales en el modo fundamental hasta que la estructura alcance ciertos límites de desplazamientos o se vuelva inestable. Se trata de un análisis suficiente para determinar la capacidad resistente última de la estructura ante acciones horizontales, aunque debe ser utilizado con cautela en estudios de vulnerabilidad sísmica cuando se trate de estructuras de fábrica (11).

Los Modelos 2 y 4 han sido discretizados utilizando elementos 3D o tetraedros de primer orden de 4 nodos e integración completa con control del bloqueo por cortante y del bloqueo volumétrico ( $\mathrm{C}_{3} \mathrm{~d} 4$ ), el modelo presenta 1.066.984 elementos, y 586.422 G.D.L, mientras que para el Modelo 3 se han utilizado elementos 2D-placa (CPe4) o elementos de cuatro nodos de integración completa con un total de 60.363 elementos y 125.262 G.D.L. El análisis se ha realizado desde el entorno de la aplicación ABAQUS 6.9-1.

\subsection{Caracterización de los materiales}

En el Modelo 1, de análisis mediante líneas de empuje, no es preciso considerar el material más que a nivel de densidades y pesos (Tabla 1). No obstante, para la estimación de la resistencia del muro perimetral se supone un material que responda a un modelo de fallo Mohr-Coulomb [1] (13), donde el valor inicial de la cohesión $f_{v k o}$ se desprecia, dado que en este tipo de estructuras el incremento de los valores de axil es muy alto desde el inicio, y $\sigma_{d}$ representa la tensión vertical de compresión en el punto considerado.

$$
f_{v k}=f_{v k o}+0.36 \sigma_{\mathrm{d}} \leq 0.065 f_{c k}
$$

Modelo 2. Para la caracterización elástica de los materiales (Tabla 1) se recurre a un análisis de algunas muestras tomadas de los tapiales de la torre que revela un gran parecido con los materiales de la Torre de Comares, también perteneciente al conjunto de la Alhambra (14). De esta otra edificación aledaña sí se dispone de ensayos y caracterizaciones mecánicas en relación al tapial calicastrado, a las piezas de ladrillo y al mortero empleado que se han tomado como referencia (5) (15).

Modelos 3 y 4. En documentos como (16) y (17), se demuestra que modelos constitutivos creados inicialmente para la simulación del hormigón no lineal son válidos para simular fábri- 
ca de ladrillo y piedra, ya que presentan un comportamiento elástico y lineal a tracción hasta la rotura, frágil en este caso, y un comportamiento elastoplástico con ablandamiento y rotura dúctil en compresión. Partiendo de estas premisas se han analizado estructuralmente edificaciones como la basílica de Santa María all'Impruneta (Italia) (18). A partir de lo anterior y de la herramienta informática utilizada en el análisis, se ha acudido al material definido según Concrete Damaged Plasticity (19) (20). Se propone, por tanto, un material inelástico con rotura frágil a tracción y dúctil a compresión, con capacidad de definir daño post-fallo. Dicho daño modifica el valor del módulo de deformación elástico según un valor escalar comprendido entre o-1. En nuestro análisis se ha definido un valor del daño tanto en tracción $\left(d_{t}\right)$ como en compresión $\left(d_{c}\right)$ de hasta un $90 \%$ de daño $\left(d_{c}=d_{t}=0,9\right)$. En el caso particular de la tracción, el daño se ha definido de manera lineal a partir de la energía de fractura, según la ecuación [2] (20).

$$
U_{t, \mathrm{o}}=\frac{2 G_{f}^{1}}{s_{t, \mathrm{o}}}
$$

La diversidad de sistemas constructivos en el interior de la torre conduce a cúpulas de distinta geometría y aparejos en cada uno de los niveles, cúpulas ejecutadas mediante fábricas con una proporción diferente de espesores de bloque de ladrillo frente a llaga de mortero. Así, se ha caracterizado la fábrica de ladrillo de cada uno de los seis niveles que constituyen la torre con un módulo de elasticidad obtenido en función de la proporción entre los espesores de bloque y llaga y de la proporción entre sus respectivos módulos de elasticidad (Tabla 2) (21).

Según la Tabla 2, el módulo de elasticidad de la fábrica puede adoptar valores dispares. Es por esto que en el tercer procedimiento de análisis, aplicado a los Modelos 3 y 4, se ha llevado a cabo un estudio de sensibilidad a partir del factor $E / E$. En la Tabla 2 se recoge el valor de este factor para los distintos niveles. Según los valores obtenidos para los diferentes niveles, el estudio de sensibilidad se realizará para un valor de $E / E_{t}$ comprendido en un rango entre 1 y 2,5 .

\section{ANÁLISIS DE RESULTADOS}

\subsection{Análisis geométrico preliminar}

El estudio de un elemento estructural a partir de líneas de empuje permite analizar el equilibrio del mismo así como establecer un coeficiente de seguridad a partir de la configuración geométrica y el peso específico de los materiales. Es
Tabla 2. Caracterizaciones de fábricas de ladrillo.

\begin{tabular}{|l|c|c|c|c|}
\cline { 2 - 5 } \multicolumn{1}{c|}{} & $\alpha\left(\mathbf{h}_{\mathbf{m}} / \mathbf{h}_{\mathbf{b}}\right)$ & $\beta\left(\mathbf{E}_{\mathbf{b}} / \mathbf{E}_{\mathbf{m}}\right)$ & $\mathbf{E} \mathbf{( M P a )}$ & $\mathbf{E} / \mathbf{E}_{\mathbf{t}}$ \\
\hline Nivel 1 & 1,20 & 12 & 1429 & 1,19 \\
\hline Nivel 2 & 0,25 & 12 & 3125 & 2,60 \\
\hline Nivel 3 & 0,50 & 12 & 2143 & 1,79 \\
\hline Nivel 4 & 0,50 & 12 & 2143 & 1,79 \\
\hline Nivel 5 (arcos y pilas) & 0,90 & 12 & 1610 & 1,34 \\
\hline Nivel 5 (cúpulas) & 0,40 & 12 & 2414 & 2,01 \\
\hline Nivel 6 & 1 & 12 & 1538 & 1,28 \\
\hline
\end{tabular}

de alcance limitado pero de utilidad en estudios preliminares que analizan el comportamiento del elemento bajo cargas gravitatorias, teniendo en cuenta, sobre todo, que se desarrolla a partir de un análisis plano sencillo.

En relación con los resultados obtenidos tras el estudio de los arcos en todos los niveles, puede reconocerse en cada uno de los casos una línea de equilibrio contenida en la sección de los mismos sin necesidad de hacer consideraciones en torno a la capacidad resistente del relleno situado en el trasdós. Los resultados presentan unos valores respecto al coeficiente geométrico de seguridad muy similar en todos los niveles, destaca el nivel 7 como el más favorable y el nivel 3 como el más desfavorable con valores del coeficiente geométrico de seguridad en los arcos principales sensiblemente por debajo de dos (Figura 2a).

En cuanto al muro perimetral, puede observarse que la configuración del nivel superior es muy determinante en la estabilidad global del elemento, situándose el coeficiente de seguridad próximo a 1,5 para una configuración de empujes como la actual (Figura 2b). Existe un punto crítico en el cruce con la última entreplanta. El desvío de la línea de empuje en el cruce con la última entreplanta, es corregido con el efecto del relleno de dicho nivel.

La composición, y por tanto la densidad, del relleno correspondiente a los distintos niveles no son conocidos con exactitud, pero la consideración de rellenos de distinta densidad no ha dado lugar a configuraciones inestables en ningún caso.

\subsection{Comportamiento a peso propio}

A partir del análisis elástico y lineal llevado a cabo sobre el Modelo 2, se obtienen, de un lado, valores de coeficientes de seguridad correspondientes a un trabajo del muro en su base

Tabla 1. Propiedades de los materiales.

\begin{tabular}{|c|c|c|c|c|c|}
\hline \multirow{2}{*}{\multicolumn{2}{|c|}{ PROPIEDADES }} & \multicolumn{2}{|c|}{ ANÁLISIS ELÁSTICO Y LINEAL } & \multicolumn{2}{|c|}{$\begin{array}{c}\text { ANÁLISIS ESTÁTICO } \\
\text { NO LINEAL }\end{array}$} \\
\hline & & Fábrica de ladrillo & Tapial & $\begin{array}{c}\text { Fábrica } \\
\text { de ladrillo }\end{array}$ & Tapial \\
\hline \multicolumn{2}{|c|}{ Densidad aparente $\left(\mathrm{kg} / \mathrm{m}^{3}\right)$} & 1450 & 2250 & 1450 & 2250 \\
\hline \multicolumn{2}{|c|}{ Módulo de elasticidad (MPa) } & $\begin{array}{l}3000 \text { (instantáneo) } \\
1600 \text { (a plazo infinito) }\end{array}$ & $\begin{array}{l}1200 \text { (estructura) } \\
6300 \text { (cimentación) } \\
\text { (al 40\% de la carga de rotura) }\end{array}$ & 1200 & 1200 \\
\hline \multicolumn{2}{|c|}{ Coeficiente de Poisson } & 0,25 & $\begin{array}{l}\text { o,3 (estructura) } \\
\text { o,2 (cimentación) }\end{array}$ & 0,2 & 0,3 \\
\hline Compresión & $\begin{array}{l}\text { Resistencia a compresión } \\
\left(f_{c}\right)(\mathrm{MPa})\end{array}$ & 4 & $\begin{array}{l}2,5 \text { (estructura) } \\
8 \text { (cimentación) }\end{array}$ & 2 & $0,5^{-}$ \\
\hline Tracción & $\begin{array}{l}\text { Resistencia a tracción }\left(f_{t}\right) \\
(\mathrm{MPa})\end{array}$ & 0,2 & $\begin{array}{l}\text { o,3 (estructura) } \\
\text { o,75 (cimentación) } \\
\text { (Relación 1/8 aprox.) }\end{array}$ & 0,2 & $0,1^{-}$ \\
\hline \multicolumn{2}{|c|}{ Energía de fractura $(\mathrm{MPa} / \mathrm{m})$} & - & - & 40 & 20 \\
\hline
\end{tabular}



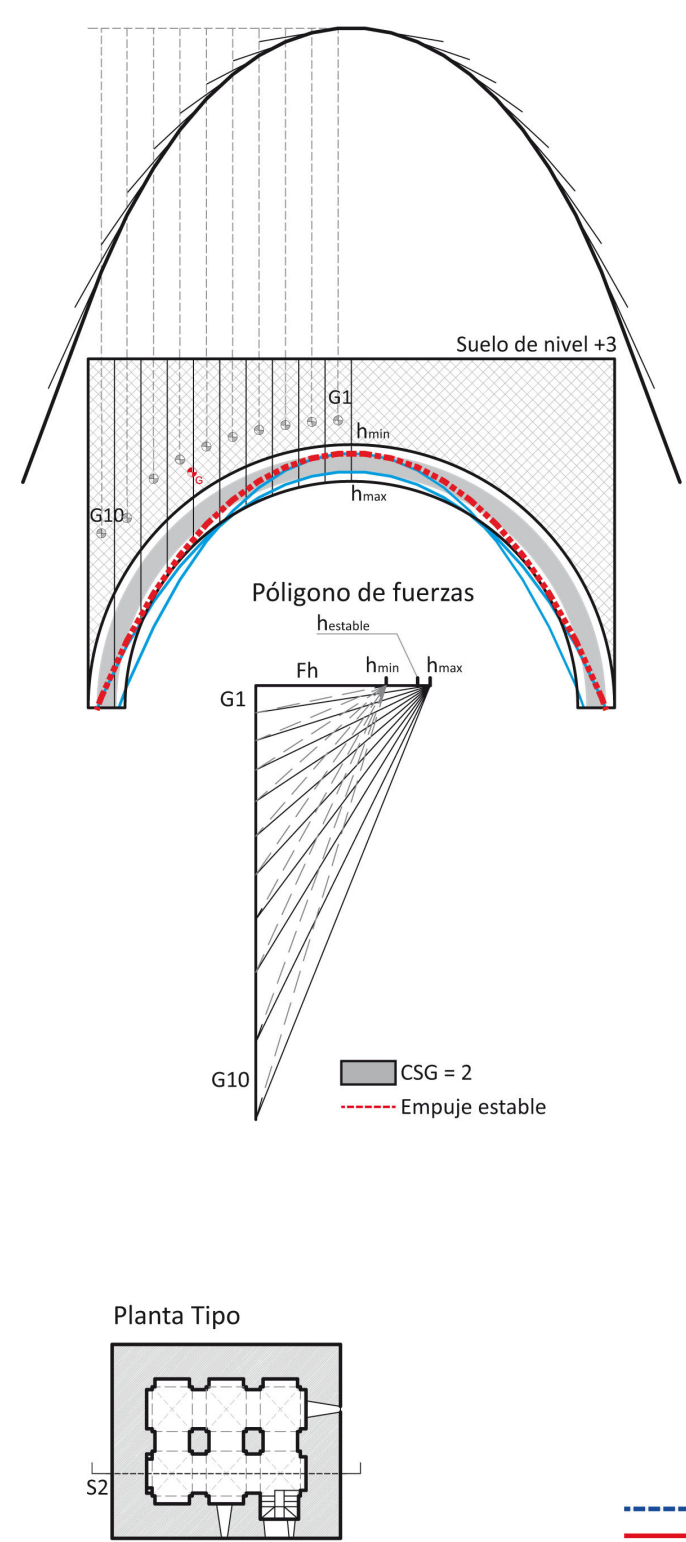

Sección S2

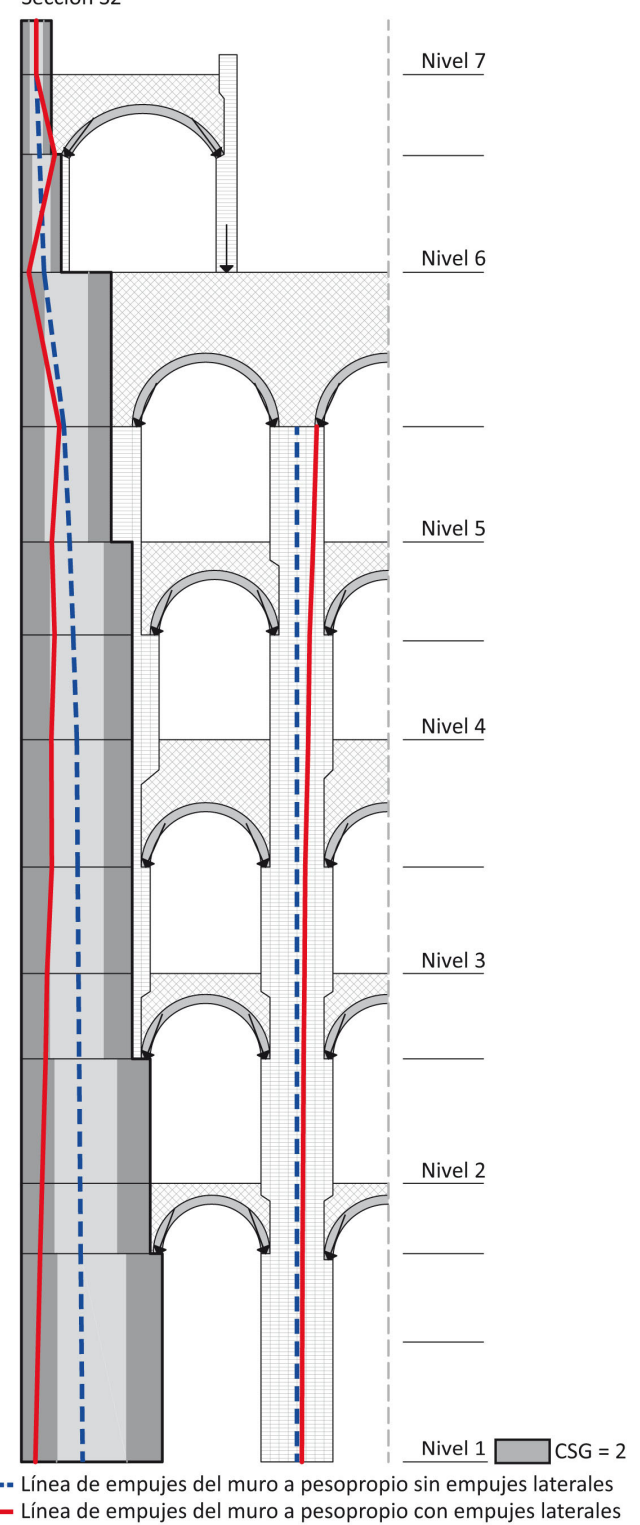

Figura 2. Análisis mediante líneas de empuje. a) Arco fajón más desfavorable (nivel 3), b) Sección este-oeste.

a compresión y a cortante. En el trabajo a compresión, dichos coeficientes de seguridad oscilan entre 4,63 para el muro de tapial (sección N-S) y 6,55 en el muro de ladrillo (sección E-O).

Por otro lado, cabe destacar que el hecho de llevar a cabo un análisis elástico y lineal como el descrito aplicado a la totalidad de la torre, conduce a una serie de concentraciones de tensiones localizadas fundamentalmente en los arranques y clave de arcos y bóvedas. Este fenómeno es más acusado en las plantas más bajas. Las tensiones de tracción alcanzan valores entre 0,25 y 0,3 $\mathrm{MPa}$, por lo que sería esperable encontrar patologías en estas zonas que, sin embargo, no existen. Esto denota que este modelo de cálculo no es válido para el tipo de análisis que nos ocupa.

Se trata de tensiones adicionales provocadas por acortamientos diferenciales excesivos entre las pilas interiores y el muro perimetral, acortamientos que suponen desplazamientos impuestos en los apoyos de arcos y cúpulas de los entrepisos. De este resultado, deriva la importancia de considerar en el análisis las distintas fases del proceso constructivo, ya que estos acortamientos se reducen y también, por tanto, las tensiones adicionales asociadas. De un análisis en el que las fases del proceso constructivo son consideradas, se obtiene un acortamiento diferencial entre los arranques de los arcos extremos de planta baja un $30 \%$ menor que el acortamiento correspondiente obtenido sin tener en cuenta dichas fases. Respecto a las tensiones principales de tracción, comentar que en los encuentros entre los arcos y el muro perimetral en planta baja se han llegado a reducir hasta un $50 \%$.

Debido a la necesidad de caracterizar correctamente las fases constructivas, según se recoge en el párrafo anterior, en los modelos de elementos finitos se han incorporado la masa y la rigidez de cada planta en diferentes escalones de carga, según la secuencia establecida en la Figura 3.

\subsection{Comportamiento global ante acciones horizontales}

Un análisis previo de determinadas condiciones de la torre colabora en el establecimiento de criterios de simplificación 

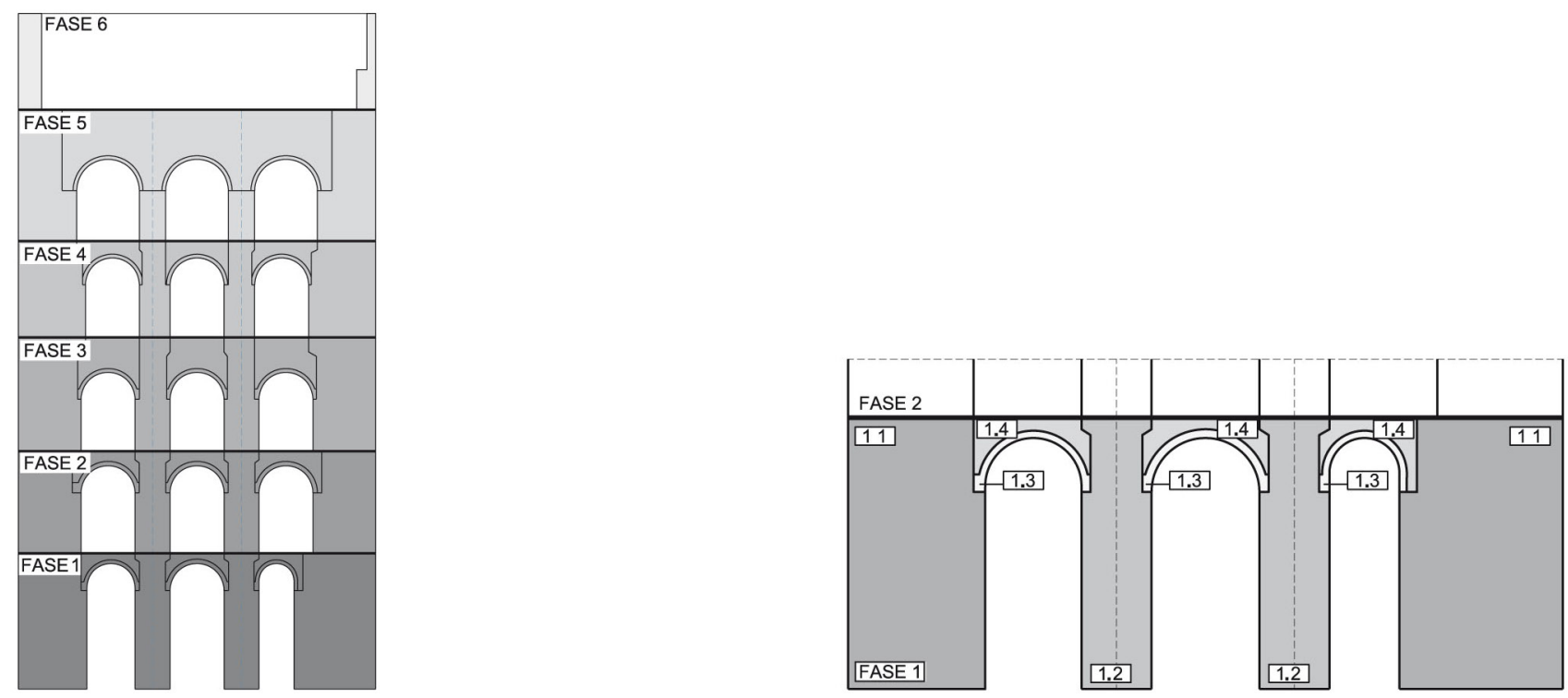

Figura 3. Fases constructivas consideradas en los modelos de elementos finitos. Dentro de cada una, se han incorporado en primer lugar masa y rigidez del muro perimetral, seguidas de las correspondientes a las pilastras interiores, a las bóvedas de ladrillo y, por último, al relleno.

del modelo a utilizar en el análisis del comportamiento ante acciones horizontales de envergadura, tales como la acción del sismo.

Un estudio de la distribución de masas del edificio puede ayudar a localizar los principales elementos resistentes. Igualmente, de un análisis modal del caso de estudio se pueden obtener la relación entre las rigideces de los pórticos interiores y los muros perimetrales.

A partir de lo anterior, puede afirmarse que la masa atribuida al tapial se acerca al $70 \%$ de la masa total. Si además se considera la capa de ladrillo interna de los muros, la masa total de los mismos alcanza valores cercanos al $75 \%$ de la masa total (Figura 4).

Por otro lado, del análisis modal se desprende que la influencia de la rigidez lateral de los entrepisos apenas contribuye entre un $5 \%$ y un $11 \%$ a la rigidez global del mismo (Tabla 3 ).

Tal como se comentó anteriormente, se desarrolla un análisis estático no lineal tipo pushover para estudiar el comporta-

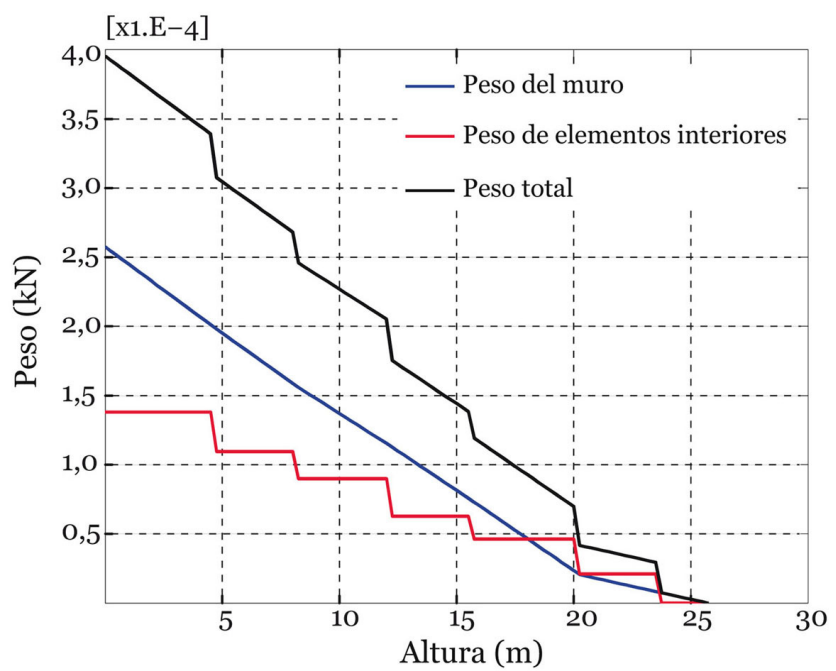

Figura 4. Distribución de pesos en altura.
Tabla 3. Análisis modal.

\begin{tabular}{|l|c|c|c|c|c|}
\hline \multirow{2}{*}{ Modo } & \multicolumn{2}{|c|}{ Con entrepisos } & \multicolumn{2}{c|}{ Sin entrepisos } & (*) \\
\cline { 2 - 6 } & $\mathbf{( s )}$ & $\mathbf{( H z )}$ & $\mathbf{( s )}$ & $\mathbf{( H z )}$ & $\mathbf{( \% )}$ \\
\hline $\begin{array}{l}\text { Modo 1. } \\
\text { Flexión E-O }\end{array}$ & 0,39 & 2,55 & 0,43 & 2,31 & $+10,2 \%$ \\
\hline $\begin{array}{l}\text { Modo 2. } \\
\text { Flexión N-S }\end{array}$ & 0,34 & 2,87 & 0,37 & 2,69 & $+6,6 \%$ \\
\hline
\end{tabular}

*Variación en el período de vibración (s) al eliminar los entrepisos en el análisis.

miento de la torre ante acciones horizontales. Al llevarlo a cabo sobre un modelo completo de la misma, el análisis detiene su evolución debido, en primer lugar, al fallo de elementos interiores. Es por esto que se analiza, por un lado, la evolución de la plastificación de los elementos interiores sobre el Modelo 3, un modelo plano basado en el pórtico central de la torre (Figura 5 a y $5 \mathrm{~b}$ ).

Las curvas de capacidad obtenidas en el análisis del Modelo 3 , muestran cómo una relación menor entre módulos de elasticidad implica que el modelo soporte menos carga, evolucionando el cálculo considerablemente menos. En todos los casos, las divergencias hacen que el análisis se detenga para unos niveles de carga considerados entre $70 \mathrm{KN}$ y $200 \mathrm{KN}$ (Figura 5c).

A la vista de las consideraciones anteriores, podemos establecer, por otro lado, un modelo de análisis de mayor simplicidad para valorar el comportamiento de la torre ante acciones horizontales. En este modelo sólo se incluyen los muros perimetrales como principales elementos resistentes.

El análisis pushover del Modelo 4, muestra, en cambio, que es menos sensible a la relación entre el módulo de deformación longitudinal del ladrillo y el del tapial (Figura 6). En una configuración tridimensional, el muro perimetral de tapial hace que la presencia del ladrillo en el muro tenga más sentido desde un punto de vista constructivo, y como soporte de los elementos del entrepiso, que desde un punto de vista estructural. En este caso, se alcanza un desplazamiento considerablemente mayor que en el Modelo 3 , llegándose a una carga de fallo en torno a $5000 \mathrm{KN}$. 


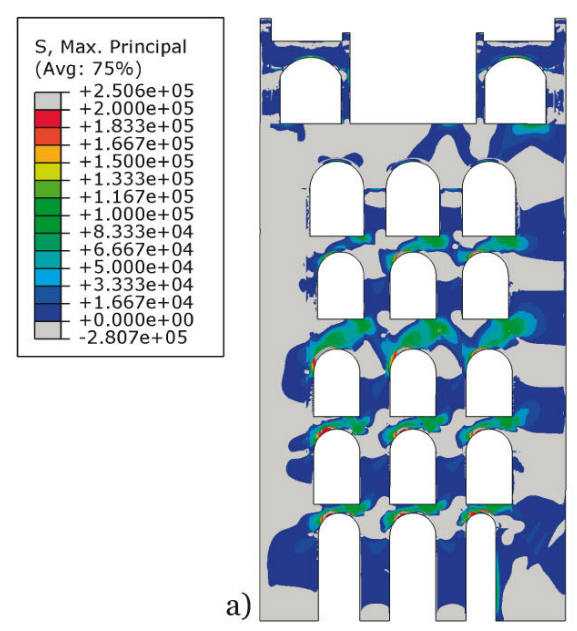

b)
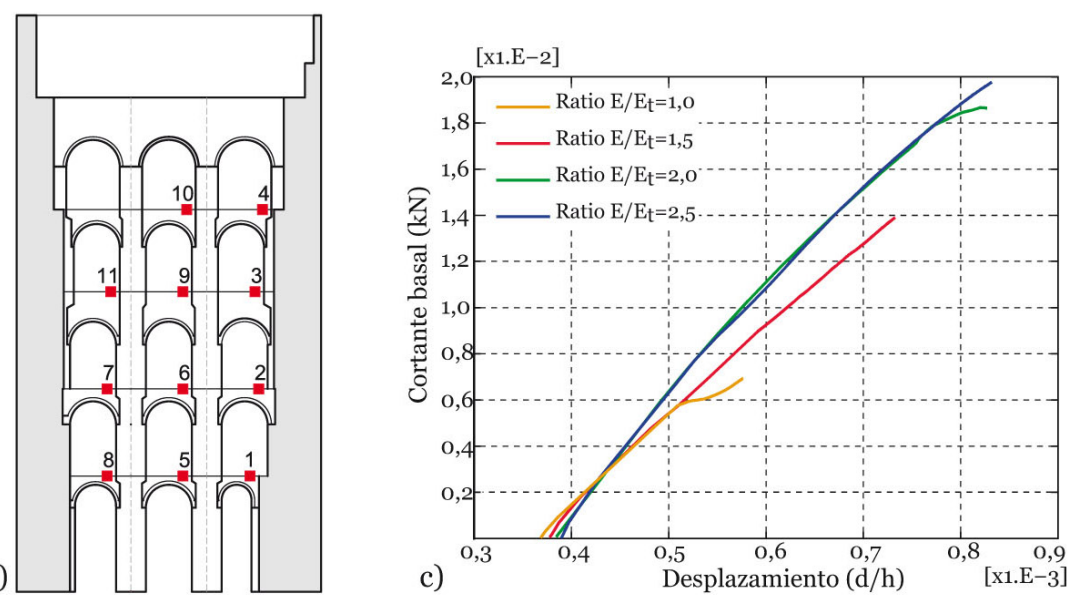

Figura 5. Análisis pushover sobre el Modelo 3. a) Tensiones principales de tracción, b) Orden de aparición de plastificaciones, c) Curvas de capacidad.

Analizando las curvas de capacidad asociadas a los casos $a$ y $b$ de la Figura 7, es decir, las curvas correspondientes a toda la base del muro o a los muros dispuestos longitudinalmente respecto a la dirección de la carga respectivamente, se comprueba que los muros perimetrales dispuestos transversalmente colaboran en la resistencia del cortante basal aproximadamente en un porcentaje del $25 \%$ del total, dada su dimensión.

Por otro lado, reconocida la proximidad entre las curvas de capacidad asociadas a los casos $d$ y $e$, (uno de los muros longitudinales como parte de la estructura de la torre y el mismo considerándolo un elemento aislado respectivamente), se deduce la validez que un análisis plano del mismo tendría para evaluar su comportamiento ante acciones horizontales.

\section{CONCLUSIONES}

- La torre presenta un buen comportamiento a peso propio basado, entre otros factores, a la configuración geométrica de la torre y de sus distintos elementos. Ante acciones horizontales, los elementos interiores son los primeros en alcanzar el fallo, constituyéndose los muros perimetrales como principales elementos resistentes. Esta estructura

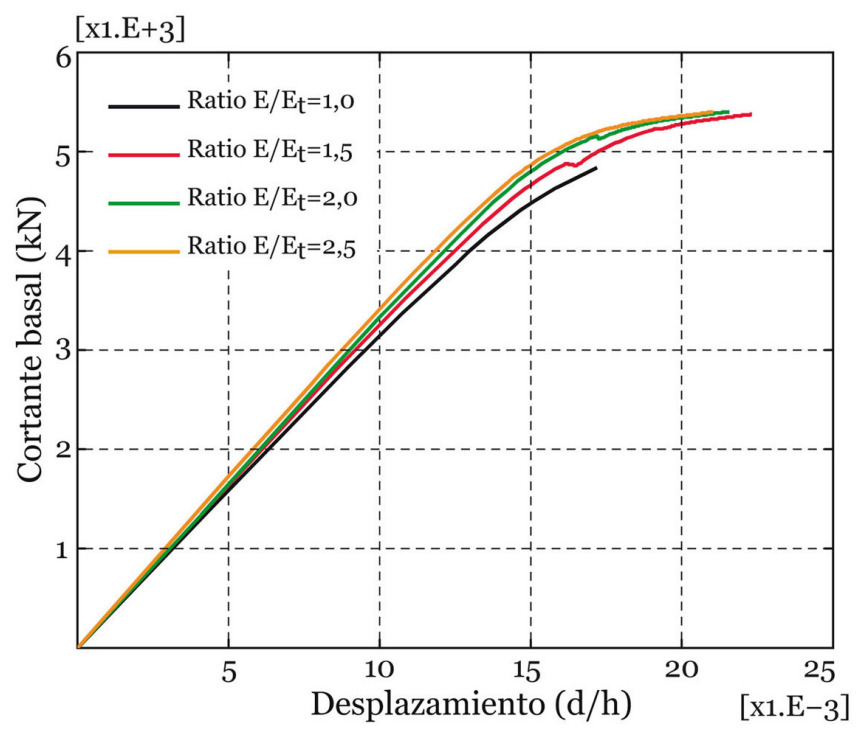

Figura 6. Análisis pushover sobre el Modelo 4. Curvas de capacidad.

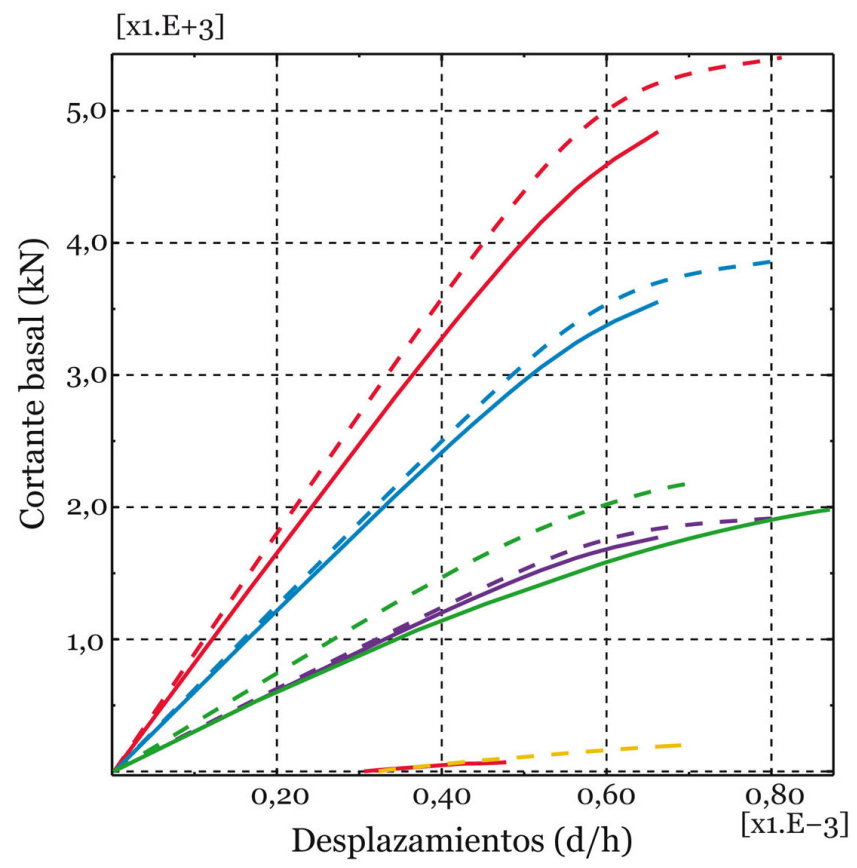

a)

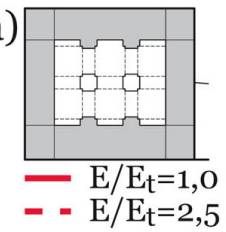

b)

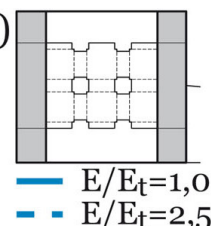

c)

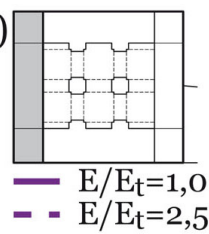

d)

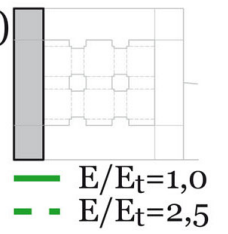

e)

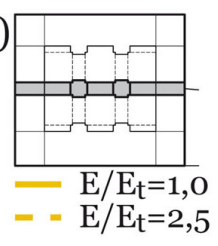

Figura 7. Curvas de capacidad correspondientes a diferentes modelos y elementos.

perimetral presenta un comportamiento global más cercano al asociado al tubo estructural que al de un sistema de pórticos.

- Es importante tener en cuenta en el análisis las distintas fases del proceso constructivo. En el caso que se estudia, no 
hacerlo conduce a estados tensionales excesivos que pueden llegar a invalidar el análisis.

- El análisis pushover del pórtico central resulta válido para evaluar el mecanismo de fallo pero no para cuantificar su resistencia última como parte del sistema global.
- Las curvas de capacidad de los modelos con una mayor relación de volumen de fábrica de ladrillo respecto a volumen de tapial son más sensibles a la relación $E / E t$.

\section{REFERENCIAS}

(1) Gallego-Burín, A. (1963). La Alhambra. Granada: Patronato de la Alhambra y el Generalife.

(2) Lourenço, P.B. (2001). Analysis of historical constructions: from thrust-lines to advanced simulations. En Historical Constructions (pp. 91-116). Guimaraes.

(3) Aroca, R. (2010). Geometría y proporción en las estructuras: Ensayos en honor de Ricardo Aroca. Madrid: Lampreave.

(4) Bermúdez, J. (1972). Alcazaba y Torres de la Alhambra. Granada: Obra Cultural de la Caja de Ahorros de Granada.

(5) CEDEX. (1995). Caracterización geotécnica de las condiciones de cimentación del conjunto monumental de la Alhambra y el Generalife (Informe técnico). Granada: Patronato de la Alhambra y Generalife.

(6) Moseley, H. (1833). On a new principle in statics, called the principle of least pressure. Philosophical Magazine, 3: 285288.

(7) Heyman, J. (1999). Teoría, historia y restauración de estructuras de fábrica: colección de ensayos. Madrid: Santiago Huerta.

(8) Block, P., Ciblac, T., Ochsendorf, J. (2006). Real-time limit analysis of vaulted masonry buildings. Computers \& Structures, 84(29-30): 1841-1852, doi: http://dx.doi.org/10.1016/j.compstruc.2006.08.002.

(9) Huerta, S. (2005). Mecánica de las bóvedas de fábrica: el enfoque del equilibrio. Informes de la Construcción, 56(496): 73-89, doi: http://dx.doi.org/10.3989/ic.2005.v57.i496.496.

(10) Pérez-Gálvez, F., Rodríguez-Liñan, C., Rubio, P. (2009). Determinación de las características mecánicas de los muros de fábrica de ladrillo en la arquitectura doméstica sevillana de los siglos XVIII y XIX. Informes de la Construcción, 61(514): 19-28, doi: http://dx.doi.org/10.3989/ic.06.001.

(11) Peña, F., Lourenço, P.B., Mendes, N., Oliveira, D.V. (2010). Numerical models for the seismic assessment of an old masonry tower. Engineering Structures, 32(5): 1466-1478, doi: http://dx.doi.org/10.1016/j.engstruct.2010.01.027.

(12) Chopra, A.K. (1995). Dynamics of structures: theory and applications to earthquake engineering. New Jersey: Prentice-Hall International.

(13) CTE. (2009). CTE-DB SE-F. Seguridad Estructural: Fábrica. Código Técnico de la Edificación.

(14) De la Torre, M.J. (1995). Estudio de los materiales de construcción en la Alhambra (Tesis doctoral). Granada: Universidad de Granada.

(15) Astiz, M.A. (1997-1998). Estudio de la seguridad estructural de la Torre de Comares. Cuadernos de la Alhambra, (3334): 115-132.

(16) William, K.J., Warnke, E.D. (1975). Constitutive model for the triaxial behaviour of concrete. En Proceedings of International Association for Bridge and Structural Engineers (IMES), 19: 1-30. Bergamo (Italy).

(17) Giordano, A., Mele, E., De Luca, A. (2002). Modelling of historical masonry structures: comparison of different approaches through a case study. Engineering Structures, 24(8): 1057-1069, doi: http://dx.doi.org/10.1016/So1410296(02)00033-0.

(18) Betti, M., Vignoli, A. (2011). Numerical assessment of the static and seismic behavior of the Basilica of Santa Maria all'Impruneta (Italy). Construction and Building Materials, 25(1): 4308-4324, doi: http://dx.doi.org/10.1016/j.conbuildmat.2010.12.028.

(19) Dassault Systèmes. (2009). Abaqus analysis user's manual. Providence (USA): Abaqus Inc.

(20) Lubliner, J., Oliver, J., Oller, S., Oñate, E. (1989). A plastic-damage model for concrete. International Journal of Solids Structures, 25(3): 299-326, doi: http://dx.doi.org/10.1016/0020-7683(89)90050-4.

(21) UIC. (1995). Code UIC 778-3: Recommandations pour l'Evaluation de la Capacite Portante des Ponts-Voutes Existants en Maconnerie et Beton. Paris: Union Internationales des Chemins de Fer. 